\title{
Optimal Strategy of Community O2O Terminal Distribution Modes Considering Order Fluctuation
}

\author{
Lu Qin ${ }^{1, *}$, Zhibin $\mathrm{Li}^{1}$, Zhiyuan Sun ${ }^{2}$ \\ ${ }^{1}$ School of Traffic and Transportation, Beijing Jiaotong University y, Haidian, Beijing, China \\ ${ }^{2}$ School of Urban Traffic, Beijing University of Technology, Beijing, China \\ qinlu@cledi.org.cn,15120848@bjtu.edu.cn,sunzhiyuan@bjut.edu.cn
}

Keywords: Community O2O, Terminal Distribution Mode, Order Fluctuation, Multi-objective Model

\begin{abstract}
With the rapid expansion of community $\mathrm{O} 2 \mathrm{O}$, the terminal distribution benefits are increasingly critical. Targeted at high costs and poor delivery benefits, this paper studies the optimal strategy of community $\mathrm{O} 2 \mathrm{O}$ terminal distribution modes based on periodicity and order fluctuation. The multi-objective model of the optimal decisionmaking of $\mathrm{O} 2 \mathrm{O}$ terminal distribution modes is constructed given the goal of the lowest distribution cost, the highest time accuracy and the highest communication quality. Besides, the distribution strategy of 'master-auxiliary distribution strategy' is put forward to find the optimal benefit under the order fluctuation, and the genetic algorithm and fuzzy optimization method are adopted to solve the problem. Through the case study, the research model and methods are verified.
\end{abstract}

\section{INTRODUCTION}

With the integration of Internet and social industries, community $\mathrm{O} 2 \mathrm{O}$ business has received increasing attention from our country. At the same time, data shows that the average cost of terminal distribution accounts $30 \%$ of total cost, and the delivery service level is equally uneven.

Researches on terminal distribution of $\mathrm{O} 2 \mathrm{O}$ business focus on the following aspects.

(1) In the study of the distribution path optimization, based on the hierarchical structure model, Aized T adopted a solution to optimize the distribution route under the congested environment with Petri method (Aized T,2014). Song studied the distribution path of various perishable food under certain demands (Song B D, 2016). Ruan Huan proposed the flexible distribution strategy by optimizing the path of terminal logistics distribution (Ruan Huan,2016).

(2) In the study of $\mathrm{O} 2 \mathrm{O}$ terminal distribution modes selection, Li Yusheng proposed joint distribution mode can optimize the allocation of social resources and improve the efficiency of distribution (Li Yusheng,2015). Wang Yanjie analysed the logistics mode and its problems of take-out $\mathrm{O} 2 \mathrm{O}$ and puts forward the logistics strategy optimization plan (Wang Yanjie,2015). You Baoqing compared the three terminal distribution modes and put forward the distribution mode optimization method of O2O supply chain management (You Baoqing, 2015).

The current studies on community $\mathrm{O} 2 \mathrm{O}$ terminal distribution focus on "macroscopic qualitative analysis of distribution mode" and "microscopic quantitative optimization of distribution path", without considering the low distribution benefits brought by order scale change and order fluctuation in a certain period.

The paper takes order scale and order fluctuation into consideration, and build the relationship between average distribution time, order quantity and labour number. On this basis, 'community $\mathrm{O} 2 \mathrm{O}$ terminal distribution decision-making model' is structured and the genetic algorithm and fuzzy optimization method are adopted to solve the model. Finally, the case study is used to verify the model validity.

\section{THE RELATIONSHIP MODEL OF TERMINAL DISTRIBUTION KEY INDICES}

\subsection{O2O Terminal Distribution Modes}

In terms of the production cost, the community $\mathrm{O} 2 \mathrm{O}$ terminal distribution can be divided into order conversion, order picking, order distribution and customer handover. Based on the trend of terminal distribution, the delivery strategy will be optimized based on different modes of each link.

(1) Order conversion link adopts self-built mode and moved-into mode.

(2) Order picking link adopts customer-picking mode, manual-picking mode and robot-picking mode.

(3) Order distribution link adopts self-built manualdistribution mode, crowdsourcing manual-distribution mode, agent manual-distribution mode, self-built robotdistribution mode and agent robot-distribution mode.

(4) Customer handover link adopts hand-in-hand mode, cabinet handover mode and agency handover mode.

\subsection{The Relationship Model}

Assume that daily order quantity is $n$, distribution labour number is $m$, The utilization rate of labours is $k$, the average actual distribution time of each order is $\bar{t}$, order combining rate is $\theta$, daily working time is $h_{0}$.

Setting promised distribution time is $t_{0}$, shortest waiting time making customers uncomfortable is $L_{0}$.

Definite: $k=1$ means utilization rate reaches saturation, then the average actual distribution time of 
each order equals shortest waiting time making customers uncomfortable, namely $\bar{t}=L_{0}$. When $k=k_{0}$, the average actual distribution time of each order equals promised distribution time, namely $\bar{t}=t_{0}$. When $k=0$, $\bar{t}=0$.

When $k \in\left[k_{0}, 1\right], \bar{t}$ increases with $k$ in interval $\left[t_{0}, L_{0}\right]$. Setting $\bar{t}$ and $k$ are quadratic. According to 'order quantity equals to distribution' principle, the relationship model of terminal distribution key indices is

$$
\bar{t}=\frac{t_{0} L_{0} \times\left(L_{0}-t_{0}\right)}{h_{0} \times \theta \times\left(t_{0}-k_{0} L_{0}\right)} \times \frac{n}{m}-\frac{k_{0} L_{0}^{2}-t_{0}^{2}}{t_{0}-k_{0} L_{0}}
$$

\section{THE OPTIMAL DECISION-MAKING MODEL OF O2O TERMINAL DISTRIBUTION MODES}

\subsection{Model assumptions}

The optimal decision-making model includes five Assumptions as follows:

(1) Freight fare per kilometre of vehicle and robot is fixed.

(2) The maximum load of one single distribution is lower than that of the vehicle or the robot.

(3) Daily order quantity is evenly distributed between 8:00 and 21:00.

(4) Order quantity distribution in one cycle exists its peak.

(5) Each delivery labour is equipped with one vehicle.

\subsection{Model structure}

\subsubsection{Target function of distribution cost rate}

The cost in order conversion link is as below:

$$
W_{1}=\alpha \times\left(\frac{C_{p}}{120}+C_{o}\right)+(1-\alpha) \times(u \times n \times \tau)
$$

Among the formula: $\alpha=1$ means adopting self-built mode and $\alpha=0$ means adopting moved-into mode. $C_{p}$ means platform construction fee. $C_{o}$ means management fee in one cycle. $u$ means average price per order. $n$ means total number of orders in one cycle. $\tau$ means platform royalty rate.

The cost in order picking link is as below:

$$
W_{2}=n \times C_{m}
$$

Among the formula: $C_{m}$ means the average packing cost per order.

The cost in order distribution link is as below:

$$
\begin{aligned}
W_{3}=\omega_{1}\left[\frac{C_{s}^{1}}{120}+m_{1} \times C_{w}^{1}+n(1-\rho) \times C_{f}^{1}+m_{1} \times C_{t}^{1}+m_{1} \frac{C_{v}^{1}}{120}+\frac{n(1-\rho) \times \bar{l}_{1} \times C_{t r}^{1}}{\theta}\right] \\
+\omega_{2}\left[\frac{C_{s}^{2}}{120}+n(1-\rho) \times C_{f}^{2}+0.2 \times n \times C_{t}^{2}\right]+\omega_{3}\left[n(1-\rho) \times C_{f}^{3}\right] \\
+\omega_{4}\left[\frac{C_{s}^{4}}{120}+m_{4} \frac{C_{v}^{4}}{120}+\frac{n(1-\rho) \times \bar{l}_{4} \times C_{t r}^{4}}{\theta}\right]+\omega_{5}\left[n(1-\rho) \times C_{f}^{5}\right]
\end{aligned}
$$

$$
\left\{\begin{array}{l}
\omega_{1}+\omega_{2}+\omega_{3}+\omega_{4}+\omega_{5}=1 \\
\omega_{1}, \omega_{2}, \omega_{3}, \omega_{4}, \omega_{5}=0,1
\end{array}\right.
$$

In 'self-built manual-distribution mode': $C_{\mathrm{s}}^{1}$ means delivery system construction fare. $m_{1}$ means labor quantity. $C_{w}^{1}$ means average wage per labor. $\rho$ means customer self-picking rate. $C_{f}^{1}$ means average commission fee per order. $C_{t}^{1}$ means unit training fee in one cycle. $C_{v}^{1}$ means average purchase cost per vehicle. $C_{t r}^{1}$ means Freight fare per kilometer. $\overline{l_{1}}$ means average delivery distance.

In 'crowdsourcing manual-distribution mode': $C_{s}^{2}$ means delivery system construction fare. $C_{f}^{2}$ means average commission fee per order. $m_{2}$ means average wage per labor. $C_{t}^{2}$ means unit training fee in one cycle. According to crowdsourcing market data, the average order delivery number of single crowdsourcing labor is 5 monthly. Then set $m_{2}=0.2 n$.

In 'agent manual-distribution mode': $C_{f}^{3}$ means average commission fee per order.

In 'self-built robot-distribution mode': $C_{s}^{4}$ means delivery system construction fare. $m_{4}$ means robot quantity. $C_{v}^{4}$ means average purchase cost per robot. $C_{t r}^{4}$ means Freight fare per kilometer. $\overline{l_{4}}$ means average delivery distance.

In 'agent robot-distribution mode': $C_{f}^{5}$ means average commission fee per order.

Because robot can't complete order handover through cabinet, assume that: in robot delivery order, $\beta$ means handover rate by adopting hand-in-hand mode, then $(1-\beta)$ means handover rate by adopting agency handover mode. Assume that: in manual delivery order, $\gamma_{1}$ means handover rate by adopting hand-in-hand mode, $\gamma_{2}$ means handover rate by adopting cabinet handover mode, $\gamma_{3}$ means handover rate by adopting agency handover mode.

The cost in customer handover link is as below:

$$
\begin{aligned}
W_{4}= & {\left[\gamma_{2} \times\left(\omega_{1}+\omega_{2}+\omega_{3}\right)\right] \times n(1-\rho) \times C_{\mathrm{sc}}^{1} } \\
& +\left[\gamma_{3} \times\left(\omega_{1}+\omega_{2}+\omega_{3}\right)+(1-\beta) \times\left(\omega_{4}+\omega_{5}\right)\right] \times n(1-\rho) \times C_{\mathrm{sc}}^{2}
\end{aligned}
$$

The constraints:

$$
\left\{\begin{array}{l}
0 \leq \beta \leq 1 \\
\gamma_{1}+\gamma_{2}+\gamma_{3}=1 \\
0 \leq \gamma_{1}, \gamma_{2}, \gamma_{3} \leq 1
\end{array}\right.
$$

Among the formula: $C_{\mathrm{sc}}^{1}$ means average storage fee of cabinet per order. $C_{\mathrm{sc}}^{2}$ means average storage fee of agency per order.

According to the cost of four order link above, target function of distribution cost rate is as below:

$$
\min \mathrm{P}=\min \left[\frac{W_{1}+W_{2}+W_{3}+W_{4}}{u \times n}\right]
$$

The constraints: 


\subsubsection{Target function of distribution time accuracy}

Assume that: $\overline{t_{k i}}$ means average distribution time at the $i^{\text {th }}$ day by adopting the $k^{\text {th }}$ distribution mode. $t_{0}$ means promised distribution time. $L_{0}$ means shortest waiting time making customers uncomfortable, and $t_{0}<L_{0}$. Then the function of distribution time accuracy is:

$$
x_{k i}= \begin{cases}1 & ; \quad \overline{t_{k i}} \leq t_{0} \\ \frac{L_{0}-\overline{t_{k i}}}{L_{0}-t_{0}} ; & t_{0}<\overline{t_{k i}}<L_{0} \\ 0 \quad ; \quad L_{0}<\overline{t_{k i}}\end{cases}
$$

(1) In 'self-built manual-distribution mode', the time accuracy at the $i^{\text {th }}$ day is as below:

$$
x_{1 i}=\frac{L_{0}-\overline{t_{1 i}}}{L_{0}-t_{0}}=\frac{-t_{0} L_{0}}{h_{1} \theta\left(t_{0}-k_{1} L_{0}\right)} \times \frac{n_{i}(1-\rho)}{m_{1}}+\frac{t_{0}}{t_{0}-k_{1} L_{0}}
$$

(2) In 'crowdsourcing manual-distribution mode', setting that the actual distribution time submits to uniform distribution function in the interval $\left[t_{0}, L_{0}\right]$, and its desired value is as below:

$$
E\left(\overline{t_{2 i}}\right)=\frac{t_{0}+L_{0}}{2}
$$

The time accuracy at the $i^{\text {th }}$ day in 'crowdsourcing manual-distribution mode' is as below:

$$
x_{2 i}=\frac{L_{0}-\overline{t_{2 i}}}{L_{0}-t_{0}}=\frac{1}{2}
$$

(3) In 'agent manual-distribution mode', setting that the actual distribution time submits to $y=-x+L_{0}$ distribution in the interval $\left[t_{0}, L_{0}\right]$, and its desired value is as below:

$$
E\left(\overline{t_{3 i}}\right)=L_{0}-\frac{\sqrt{2}}{2}\left(L_{0}-t_{0}\right)
$$

The time accuracy at the $i^{\text {th }}$ day in 'agent manualdistribution mode' is as below:

$$
x_{3 i}=\frac{L_{0}-\overline{t_{3 i}}}{L_{0}-t_{0}}=\frac{\sqrt{2}}{2}
$$

(4) In 'self-built robot-distribution mode', assuming that: daily working time each robot is $h_{4}$. The optimal time accuracy of distribution robot utilization is $k_{4}$. The time accuracy at the $i^{\text {th }}$ day in 'self-built robotdistribution mode' is as below:

$$
x_{4 i}=\frac{L_{0}-\overline{t_{4 i}}}{L_{0}-t_{0}}=\frac{-t_{0} L_{0}}{h_{4} \theta\left(t_{0}-k_{4} L_{0}\right)} \times \frac{n_{i}(1-\rho)}{m_{4}}+\frac{t_{0}}{t_{0}-k_{4} L_{0}}
$$

(5) In 'agent robot-distribution mode', under the accurate forecast in future, distribution time will almost equal to the promised time, that means $\overline{t_{5 i}}=t_{0}$. The time accuracy at the $i^{\text {th }}$ day in 'agent robot-distribution mode' is as below:

$$
x_{5 i}=1
$$

Using the mean value of the time accuracy in one cycle as the measure index, the function of distribution time accuracy is as below:

$$
\operatorname{maxT}=\max \frac{1}{z}\left(\omega_{1} \sum_{i=1}^{2} x_{1 i}+\omega_{2} \sum_{i=1}^{2} x_{2 i}+\omega_{3} \sum_{i=1}^{2} x_{3 i}+\omega_{4} \sum_{i=1}^{2} x_{4 i}+\omega_{5} \sum_{i=1}^{2} x_{5 i}\right)
$$

Among that: $z=30$ is used to calculate for master strategy, and $\mathrm{z}=$ 'the number of order peak' is used to calculate for auxiliary strategy.

\subsubsection{Target function of communication quality}

When communication body is human, the communication quality is determined by 'training effect' and 'training cost'. Assume that $C_{t}^{j}$ means training expense per capita under the $j^{\text {th }}$ handover mode. $e_{t}^{j}$ means training effect under the $j^{\text {th }}$ handover mode and $e_{t}^{j} \in[0,1] . C_{t}$ means training expense per capita making customer satisfied.

Definite: When $0<a<1,\langle a\rangle=a$. When $a \geq 1,\langle a\rangle=1$. The formula of communication quality is:

$$
x_{j}^{\prime}=\left\langle\frac{e_{t}^{j} C_{t}^{j}}{C_{t}}\right\rangle
$$

The communication quality in 'self-built manualdistribution mode' is as below:

$$
x_{1}^{\prime}=\left\langle\frac{e_{t}^{1} C_{t}^{1}}{C_{t}}\right\rangle
$$

The communication quality in 'crowdsourcing manual-distribution mode' is as below:

$$
x_{2}^{\prime}=\left\langle\frac{e_{t}^{2} C_{t}^{2}}{C_{t}}\right\rangle
$$

the Communication quality in 'agent manualdistribution mode' is as below:

$$
x_{3}^{\prime}=\left\langle\frac{e_{t}^{3} \times b \times C_{f}^{3}}{C_{t}}\right\rangle
$$

Among that: Assuming per capita training cost is proportional to the distribution cost of a single order. That means $C_{t}^{3}=b \times C_{f}^{3}$.

When communication body is robot or cabinet, there exists a standard handover process, and it can be regarded as the value of communication quality is highest. Then the communication quality value in 'selfbuilt robot-distribution mode', 'agent robot-distribution mode' and 'cabinet handover mode' are as below:

$$
x_{4}^{\prime}=x_{5}^{\prime}=x_{7}^{\prime}=1
$$

Because robot can't complete order handover through cabinet, adopting 'cabinet handover mode' by robot should be removed.

Set $o_{1}=\gamma_{1} \times \omega_{1} \times x_{1}^{\prime} . o_{1}$ is communication quality value by adopting 'self-built manual-distribution mode' and 'hand-in-hand mode'.

Set $o_{2}=\gamma_{1} \times \omega_{2} \times x_{2}^{\prime} . o_{2}$ is communication quality value by adopting 'crowdsourcing manual-distribution 
mode' and 'hand-in-hand mode'.

Set $o_{3}=\gamma_{1} \times \omega_{3} \times x_{3}^{\prime} . o_{3}$ is communication quality value by adopting 'agent manual-distribution mode' and 'hand-in-hand mode'.

Set $o_{4}=\beta \times \omega_{4} \times x_{4}^{\prime} . o_{4}$ is communication quality value by adopting 'self-built robot-distribution mode' and 'hand-in-hand mode'.

Set $o_{5}=\beta \times \omega_{5} \times x_{5}^{\prime} . o_{5}$ is communication quality value by adopting 'agent robot-distribution mode' and 'hand-in-hand mode'.

Set $o_{6}=\left[(1-\beta) \times\left(\omega_{4}+\omega_{5}\right)+\gamma_{3} \times\left(\omega_{1}+\omega_{2}+\omega_{3}\right)\right] \times x_{6}^{\prime} \quad . \quad o_{6} \quad$ is communication quality value by adopting 'agency handover mode' under robot and human distribution.

Set $o_{7}=\gamma_{2} \times\left(\omega_{1}+\omega_{2}+\omega_{3}\right) \times x_{7}^{\prime} \cdot o_{7}$ is communication quality value by adopting 'cabinet handover mode' under human distribution. below:

The target function of communication quality is as

$$
\max F=\max \sum_{i=1}^{7} o_{i}
$$

\subsubsection{The optimal decision-making model}

Based on the three target functions above, the optimal decision-making model is as below:

$$
\begin{array}{r}
\min \mathrm{P}=\min \left[\frac{W_{1}+W_{2}+W_{3}+W_{4}}{u \times n}\right] \\
\operatorname{maxT}=\max \frac{1}{Z}\left(\omega_{1} \sum_{i=1}^{z} x_{1 i}+\omega_{2} \sum_{i=1}^{2} x_{2 i}+\omega_{3} \sum_{i=1}^{2} x_{3 i}+\omega_{4} \sum_{i=1}^{2} x_{4 i}+\omega_{5} \sum_{i=1}^{2} x_{5 i}\right) \\
\max F=\max \sum_{i=1}^{7} o_{i}
\end{array}
$$

The constraint:

$$
\left\{\begin{array}{l}
\omega_{1}+\omega_{2}+\omega_{3}+\omega_{4}+\omega_{5}=1 \\
\alpha, \omega_{1}, \omega_{2}, \omega_{3}, \omega_{4}, \omega_{5}=0,1 \\
z=30, \text { the number of order peak } \\
0 \leq \beta \leq 1 \\
\gamma_{1}+\gamma_{2}+\gamma_{3}=1 \\
0 \leq \gamma_{1}, \gamma_{2}, \gamma_{3} \leq 1
\end{array}\right.
$$

\section{METHODOLOGIES}

\subsection{Algorithm selection}

Step 1 genetic algorithm

Step 1.1 Code. Use binary encoding and the number of chromosomes is 10 . Randomly generate the initial population.

Step 1.2 Calculate the fitness value of the individual. The three objective functions are used as the fitness function of the individual.

Step 1.3 Intersection. The intersection probability is set to 0.9 . 0.05 ;

Step 1.4 Mutation. The mutation probability is set to

Step 1.5 The next generation selection. Adopt the strategy of non-dominated solution and calculation of crowding degree to select the next generation. nondominated solution is used to filter the optimal solution of the subproject and calculation of crowding degree is used to filter more decentralized noncommutative solutions.

Step 1.6 Repeat from step 1.2 to step 1.5, until producing the pareto optimal solution or reaching the maximum iterations. Set the number of iterations to 100 times.

Step 2 Fuzzy optimization method

Step 2.1 Establish the relative merit matrix.

Step 2.2 Solve the relative merit of each object and confirm the optimal solution.

\subsection{Model solving steps}

To solve the problem of order fluctuation, the'masterauxiliary' distribution strategy is used for order delivery. The master distribution strategy is used for whole cycle order delivery and the auxiliary distribution strategy is used for the peak order delivery.

(1) The master distribution strategy

Step1: take 30 days as one cycle and calculate the average numbers of off-peak order. multiply it by 30 to get the total order number as $\overline{n_{F}} \times 30$. Put the total number into the model and solve the model.

Step2: Put the total order number into the model and use genetic algorithm to seek all Pareto optimal value of distribution cost rate, distribution time accuracy and communication quality.

Step3: Determine the weight of the three indicators. Use Fuzzy optimization method to find the optimal mode as the master mode.

(2) The auxiliary distribution strategy

Step1: Find all peak orders fluctuations and calculate the total order fluctuation number in the whole cycle.

Step2: Put the total peak-order fluctuation number of the whole cycle into the model. Use genetic algorithm to seek all Pareto optimal value of distribution cost rate, distribution time accuracy and communication quality.

Step3: Determine the weight of the three indicators. Use Fuzzy optimization method to find the optimal mode as the master mode.

\section{CASE STUDY}

'Company A' is a community O2O retailer. Put its orders data of three stages into the model and solve it. Get the Pareto optimal solution as below:

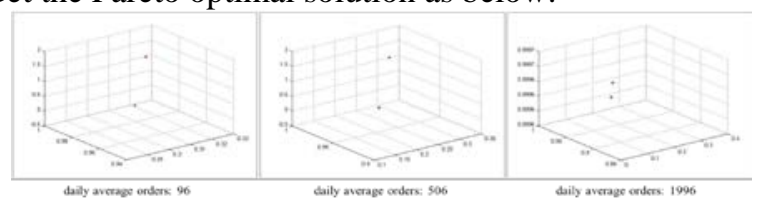

a: Off-peak Pareto optimal solutions

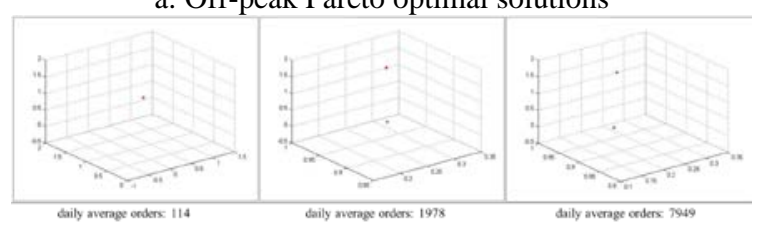

b: Peak Pareto optimal solutions

Figure 1: Pareto optimal solutions of three stages 
Based on the Pareto optimal solutions, adopt Fuzzy optimization method to find optimal result as below:

Table 1 Off-peak and peak optimal mode result

\begin{tabular}{|c|c|c|c|c|c|c|c|c|c|c|c|c|c|}
\hline \multirow{2}{*}{$\begin{array}{c}\text { Off-peak } \\
\text { average daily } \\
\text { orders }\end{array}$} & \multicolumn{10}{|c|}{ Key variable value } & \multicolumn{3}{|c|}{ Target function value } \\
\hline & $\alpha$ & $\beta$ & $w_{1}$ & $w_{2}$ & $w_{3}$ & $w_{4}$ & $w_{5}$ & $\gamma_{1}$ & $\gamma_{2}$ & $\gamma_{3}$ & $P$ & $T$ & $F$ \\
\hline 96 & 0 & 0.979 & 0 & 0 & 0 & 0 & 1 & 0.397 & 0.271 & 0.332 & 0.327 & 1 & 0.9935 \\
\hline 505 & 0 & 0.997 & 0 & 0 & 0 & 1 & 0 & 0.417 & 0.322 & 0.261 & 0.133 & 0.912 & 0.9991 \\
\hline 1996 & 1 & 0.999 & 0 & 0 & 0 & 1 & 0 & 0.426 & 0.458 & 0.116 & 0.050 & 0.890 & 0.9997 \\
\hline $\begin{array}{c}\text { Peak average } \\
\text { daily orders }\end{array}$ & $\alpha$ & $\beta$ & $w_{1}$ & $w_{2}$ & $w_{3}$ & $w_{4}$ & $w_{5}$ & $\gamma_{1}$ & $\gamma_{2}$ & $\gamma_{3}$ & $P$ & $T$ & $F$ \\
\hline 114 & 0 & 0.948 & 0 & 0 & 0 & 0 & 1 & 0.257 & 0.324 & 0.419 & 0.328 & 1 & 0.9844 \\
\hline 1978 & 0 & 0.998 & 0 & 0 & 0 & 1 & 0 & 0.395 & 0.512 & 0.093 & 0.189 & 0.864 & 0.9993 \\
\hline 7949 & 1 & 0.990 & 0 & 0 & 0 & 1 & 0 & 0.079 & 0.643 & 0.277 & 0.103 & 0.820 & 0.9971 \\
\hline
\end{tabular}

According the result above, the terminal distribution optimal strategy of 'Company A' at three stages is as follows.

(1) The strategy at first stage: the master mode for off-peak orders is agent robot-distribution mode and the auxiliary mode for peak orders is agent robot-distribution mode.

(2) The strategy at second stage: the master mode for off-peak orders is self-built robot-distribution mode and the auxiliary mode for peak orders is self-built robotdistribution mode.

(3) The strategy at second stage: the master mode for off-peak orders is self-built robot-distribution mode and the auxiliary mode for peak orders is self-built robotdistribution mode.

\section{CONCLUSIONS}

In the study of optimal strategy of community $\mathrm{O} 2 \mathrm{O}$ terminal distribution modes, order fluctuation is considered into the model. Besides, the 'master-auxiliary distribution strategy' is built to solve order fluctuation factor, which will make the model much more possible to be applied for actual distribution mode selection.
In community $\mathrm{O} 2 \mathrm{O}$ terminal distribution field, there are still the following issues worthy to further study: (1) Study the community $\mathrm{O} 2 \mathrm{O}$ terminal distribution strategy based on orders fluctuation within 24 hours. (2) study the $\mathrm{O} 2 \mathrm{O}$ end distribution strategy for communities based on fresh commodity and service commodity.

\section{REFERENCES}

[1] Aized T, Srai J., 2014. Hierarchical modelling of Last Mile logistic distribution system. 70(5-8):1053-1061.

[2] Li Yusheng., 2015. Logistics distribution mode selection of traditional retail industry under $\mathrm{O} 2 \mathrm{O}$ mode.

[3] Ruan Huan, Gen Liang, Xiao Renbin., 2016. Research on terminal logistics flexible distribution strategy based on extreme value-ant colony algorithm. 19(01):51-60.

[4] Song B D, Ko Y D., 2016. A vehicle routing problem of both refrigerated and general-type vehicles for perishable food products delivery. 169: 61-71.

[5] Wan Yanjie., 2015. Logistics strategy in takeaway O2O Market. 34(10): 23-25.

[6] You Baoqin., 2017. The optimization of logistics distribution mode in O2O supply chain management. (10): 7981. 